\title{
Hyperphosphatemic familial tumoral calcinosis caused by a mutation in GALNT3 in a European kindred
}

Received: 3 December 2005/ Accepted: 10 January 2006/Published online: 10 March 2006

(C) The Japan Society of Human Genetics and Springer-Verlag 2006

\begin{abstract}
Hyperphosphatemic familial tumoral calcinosis (HFTC) is an autosomal recessive metabolic disorder characterized by extensive phenotypic and genetic heterogeneity. HFTC was shown recently to result from mutations in two genes: GALNT3, coding for a glycosyltransferase responsible for initiating O-glycosylation, and $F G F 23$, coding for a potent phosphaturic protein. All GALNT3 mutations reported so far have been identified in patients of either Middle Eastern or African-American extraction, corroborating numerous historical reports of the disorder in Africa and in the Middle East. In the present study, we describe a patient of Northern European origin displaying typical features of HFTC. Mutation analysis revealed that this patient carries a homozygous novel nonsense mutation in GALNT3 predicted to result in the synthesis of a significantly truncated protein. The present results expand the spectrum of known mutations in GALNT3 and demonstrate the existence of HFTC-causing mutations in this gene outside the Middle Eastern and AfricanAmerican populations.
\end{abstract}

P. Specktor $\cdot$ M. Indelman · E. Sprecher $(\bowtie)$

Department of Dermatology,

Laboratory of Molecular Dermatology,

Rambam Medical Center, POB 9602, 31096 Haifa, Israel

E-mail: e sprecher@rambam.health.gov.il

Tel.: +972-4-8541919

Fax: +972-4-8542951

E. Sprecher

The Rappaport Family Institute for Research in the

Medical Sciences, 31096 Haifa, Israel

P. Specktor · E. Sprecher

Bruce Rappaport Faculty of Medicine,

Technion-Israel Institute of Technology,

Haifa, Israel

J.G. Cooper

Department of Medicine,

Stavanger University Hospital,

Stavanger, Norway
Keywords Calcinosis - Glycosylation ·

Familial $\cdot$ Mutation $\cdot$ Consanguinity

\section{Introduction}

Familial tumoral calcinosis (FTC) is a severe metabolic disorder characterized by extraosseous calcium phosphate deposition in the skin, muscle, joints, and visceral organs, resulting in incapacitating joint pain, secondary skin infections and various organ dysfunction (Metzker et al. 1988). It is often associated with hyperphosphatemia (Smack et al. 1996), and is then termed hyperphosphatemic familial tumoral calcinosis (HFTC; MIM211900). HFTC has been shown to result from loss-of-function mutations in at least two genes: $G A L$ $N T 3$ coding for UDP- $N$-acetyl-alpha-D-galactosamine: polypeptide $n$-acetylgalactosaminyltransferase 3 (ppGalNacT3) (Topaz et al. 2004; Ichikawa et al. 2005), a glycosyltransferase, which initiates O-glycosylation (Ten Hagen et al. 2003); and FGF23 (Benet-Pagès et al. 2005; Larsson et al. 2005; Araya et al. 2005; Chefetz et al. 2005) coding for fibroblast growth factor 23 (FGF23), a potent phosphaturic protein (Berndt et al. 2005).

Interestingly, mutations in GALNT3 have been identified so far only in patients of Middle Eastern or African-American origin. This corroborates previous historical clinical reports pointing to the prevalence of FTC in these regions (McClatchie and Bremner 1969; Hawass et al. 1988; Jain 1989). The existence of various trade routes between Africa and the Middle East during many centuries has been invoked to explain the peculiar geographical distribution of FTC. However, different HFTC-causing mutations have been identified in African-American and Middle Eastern patients (Topaz et al. 2004). In the present report, we describe the identification of a novel GALNT3 mutation in a patient of European extraction with features typical of HFTC, demonstrating the existence of GALNT3 mutations outside the Middle Eastern and African populations. 


\section{Materials and methods}

Patient and control individuals

Blood samples were collected after having obtained written consent from each participant according to a protocol reviewed and approved by the local Helsinki Committee and by the Israeli Ministry of Health. Genomic DNA was isolated from blood samples using the salt chloroform extraction method.

\section{Mutational analysis}

All exons and exon-intron boundaries of the GALNT3 and $F G F 23$ genes were PCR-amplified as previously described (Frishberg et al. 2005; Benet-Pagès et al. 2005).

To verify Q592X, a 496 bp PCR fragment encompassing exon 9 was amplified using primers 5'GGCTATTGTATCGTCTATCAC-3' and 5'-GATATA TTCTCTTATCACATGGG-3', and digested in the presence of $\mathrm{Xba \textrm {I }}$.

\section{Results}

Clinical findings

A 32-year-old male individual of Northern European descent was referred with hyperphosphatemia and periarticular calcification. His parents are unaware of any familial relationship. His parents and his healthy brother have normal calcium, phosphate and PTH levels.

Since childhood, this patient has suffered from recurrent episodes of conjunctival irritation and arthralgia. He damaged two front teeth in an accident when he was 9 years old. The dentist's attempt to root fill the teeth was unsuccessful due to obliterated pulp cavities. Later assessment of the patient revealed thin dental enamel, short blunt roots, taurodontism of the pre-molar/molar teeth and obliteration of the pulp cavities in most teeth (Fig. 1a).

Radiological examination revealed periarticular calcifications near the acromio-clavicular joints and both elbow joints (Fig. 1b). Ophthalmological examination disclosed whitish "salt-like" deposits on the conjunctiva as previously described (Chefetz et al. 2005). Of note, no cutaneous calcifications were observed.

Laboratory work-up disclosed elevated serum phosphate $(1.9-2.18 \mathrm{mmol} / \mathrm{l})$, normal or slightly raised serum calcium (2.5-2.8 mmol/1), normal ionized calcium (1.30$1.32 \mathrm{mmol} / \mathrm{l})$ and PTH (3.5-5.1 pmol/l) levels (Immulite 2000 Intact PTH; Diagnostic Products, Los Angeles, CA; ref: $0.7-5.7 \mathrm{pmol} / \mathrm{l})$. Measured $24 \mathrm{~h}$ creatinine clearance and calcium excretion were normal. Phosphate excretion was reduced $(6 \mathrm{mmol} / 24 \mathrm{~h})$, phosphate clearance was $2.51 \mathrm{ml} / \mathrm{min}$ yielding a fractional renal tubular
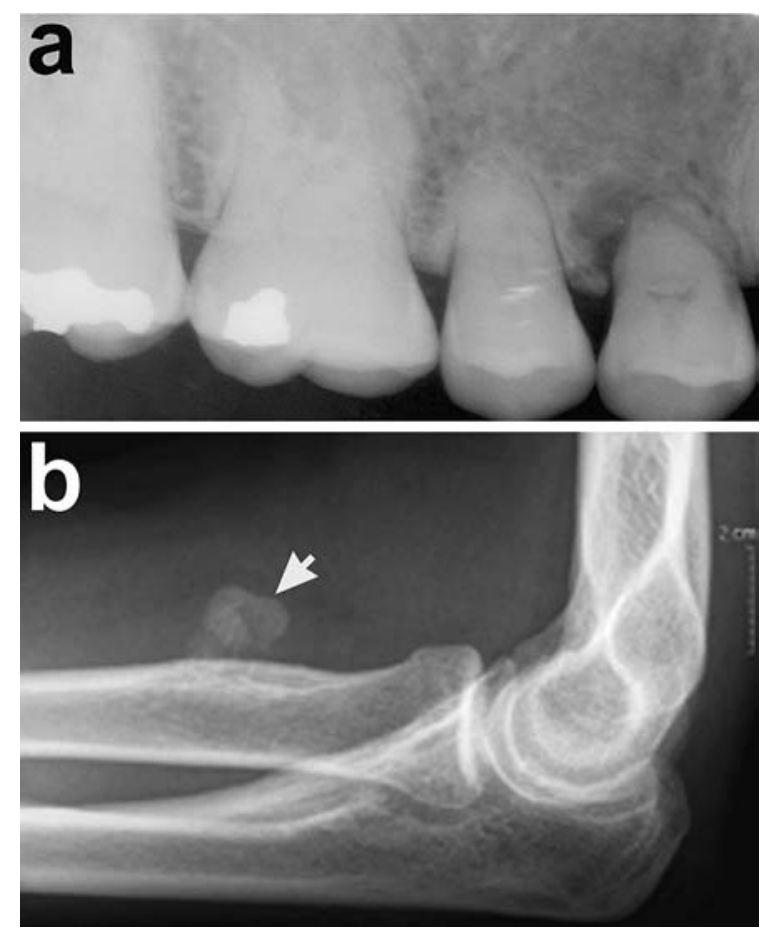

Fig. 1a,b Clinical features. a Dental radiograph demonstrating sclerotic teeth with blunt roots and obliterated pulp cavity. b Radiographs of the left elbow disclosing large calcifications

reabsorption of phosphate of $98 \%$ (normal $82-90 \%$ ); $1.25-(\mathrm{OH})_{2} \mathrm{D}(106 \mathrm{pmol} / \mathrm{l}$, ref: $50-145 \mathrm{pmol} / \mathrm{l})$ and $25-$ $(\mathrm{OH})-\mathrm{D}(45.1 \mathrm{nmol} / \mathrm{l}$, ref: $30-150 \mathrm{nmol} / \mathrm{l})$ were normal. Phosphate-restricted diet and antacids (but not treatment with sevelamer hydrochloride) for 6 months lowered phosphate levels to high normal levels without alleviating clinical signs.

\section{Mutation analysis}

Since GALNT3 mutations had never previously been described in European patients, we initially established the entire coding sequence of $F G F 23$ in this patient. No deleterious sequence alterations in FGF23 were identified.

We then sequenced the ten coding exons of GALNT3 in the patient. We identified a $\mathrm{C}>\mathrm{T}$ transition at position 1,774 of the cDNA sequence (starting from ATG). This mutation was found to be carried in a heterozygous state by his parents (Fig. 2a). Using a PCR-RFLP assay, we confirmed segregation of the mutation in the family (Fig. 2b). We also excluded the mutation from a pool of 124 chromosomes derived from healthy unaffected individuals.

The mutation is predicted to result in the substitution of a stop codon for a glutamine residue at position 592 of the ppGalNacT3 amino acid sequence (Q592X) and thus to result in the synthesis of a truncated protein lacking a significant part of its carbohydrate-binding domain (Fig. 2c). 
a

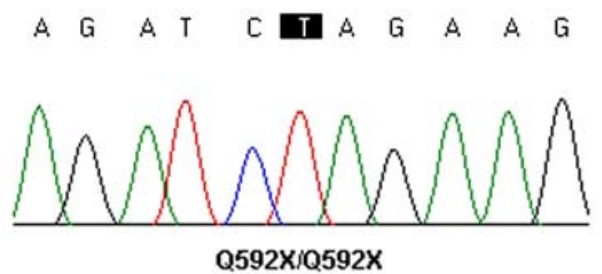

A. G A T C C C A G A A

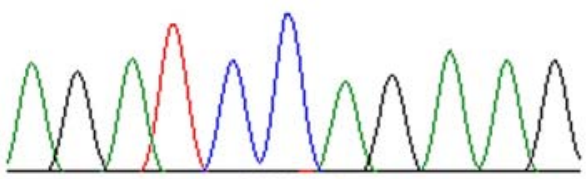

WMNT

Fig. 2a-c Mutation analysis. a Direct sequencing reveals a homozygous $\mathrm{C} \rightarrow \mathrm{T}$ transition at position 1,774 of the GALNT3 cDNA sequence (upper panel). The wildtype sequence is given for comparison (lower panel). b To confirm C1774T, a 496 bp fragment encompassing GALNT3 exon 9 was PCR-amplified and digested with $X b a \mathrm{I}$ endonuclease. Since $\mathrm{C} 1774 \mathrm{~T}$ creates a recognition site for $X b a \mathrm{I}$ endonuclease, the patient $(P)$ displays PCR fragments of

\section{Discussion}

In the present study, we report the first case of HFTC caused by a mutation in GALNT3 in a patient of nonAfrican or non-Middle-Eastern origin. This finding suggests that this disease may be more common and more widely distributed than initially thought.

Hyperphosphatemic familial tumoral calcinosis is characterized by marked phenotypic heterogeneity. Although most patients display periarticular calcifications, other manifestations, such as visceral or mucosal involvement, are not invariably seen (Gal et al. 1994; Topaz et al. 2004; Chefetz et al. 2005). Apart from severe joint disease, the present case was remarkable for prominent dental anomalies. Dental abnormalities in HFTC patients have been documented in a number of previous reports and have been found to be associated with mutations in both GALNT3 and FGF23 (Ichikawa et al. 2005; Benet-Pages et al. 2005). Some investigators have even suggested that dental findings, including short bulbous roots and obliteration of the pulp cavities as seen in the present case, may serve as a useful phenotypic marker for FTC (Burkes et al. 1991).

GALNT3 codes for the widely expressed ppGalNacT3 glycosyltransferase (Ten Hagen et al. 2003). The mechanisms underlying the role of ppGalNacT3 in maintaining phosphate homeostasis are still elusive. However, the fact that mutations in GALNT3 and in FGF23 result in a similar phenotype (Topaz et al. 2004; Benet-Pagès et al. 2005), and the fact that FGF23 levels are perturbed in patients with
$323 \mathrm{bp}$ and $173 \mathrm{bp}$, healthy individuals (C1-C4) display an undigested $496 \mathrm{bp}$ fragment only, whereas the mother $(M)$ and father $(F)$ of the patient display all fragment types. $\mathbf{c}$ The mutation results in the substitution of a stop codon for a glutamine residue at position 592 as indicated along a scheme of the ppGalNacT3 molecule. The lighter shaded area corresponds to the truncated part of the enzyme
ppGalNacT3 deficiency (Topaz et al. 2004) indicate that the two proteins participate in a common regulatory pathway. FGF23 decreases circulating phosphate levels by downregulation of NaPiIIa (Shimada et al. 2005), the major phosphate transporter in the renal proximal tubule, by inhibition of NaPiIIb, responsible for trans-intestinal phosphate transport (Miyamoto et al. 2005), and by down-regulation of 1-alpha-hydroxylation of 25-hydroxycholecalciferol (Inoue et al. 2005). Proteolytic degradation of FGF23 is believed to play a major role in regulating these activities (Saito et al. 2003). However, recent data suggest that FGF23 is also subject to O-glycosylation (Fukumoto 2005), and it is therefore tempting to speculate that ppGalNacT3-mediated O-glycosylation may be involved in the regulation of FGF23 activity. Other potential targets for ppGalNacT3-mediated O-glycosylation include FGF23 putative receptors (Yu et al. 2005) and phosphate transporters (Berndt et al. 2005).

To summarize, we have described a novel mutation in GALNT3 causing HFTC in a patient of European origin. These findings add to the expanding spectrum of genetic alterations in this gene and are in line with a growing number of reports pointing to the role played by impaired glycosylation in the pathogenesis of genetic diseases (Vogt et al. 2005).

Acknowledgements We acknowledge the family for having participated in the present study. We are grateful to V. Friedman for DNA sequencing services. This study was supported in part by grants provided by the Israel Science Foundation and the Rappaport Family Institute for Research in the Medical Sciences. 


\section{References}

Araya K, Fukumoto S, Backenroth R, Takeuchi Y, Nakayama K, Ito N, Yoshii N, Yamazaki Y, Yamashita T, Silver J, Igarashi T, Fujita T (2005) A novel mutation in fibroblast growth factor 23 gene as a cause of tumoral calcinosis. J Clin Endocrinol Metab 90:5523-5527

Benet-Pagès A, Orlik P, Strom TM, Lorenz-Depiereux B (2005) An FGF-23 missense mutation causes familial tumoral calcinosis with hyperphosphatemia. Hum Mol Genet 14:385390

Berndt TJ, Schiavi S, Kumar R (2005) Phosphatonins and the regulation of phosphorus homeostasis. Am J Physiol Renal Physiol 289:F1170-1182

Burkes EJ Jr, Lyles KW, Dolan EA, Giammara B, Hanker J (1991) Dental lesions in tumoral calcinosis. J Oral Pathol Med 20:222227

Chefetz I, Heller R, Galli-Tsinopoulou A, Richard G, Wollnik B, Indelman M, Koerber F, Topaz O, Bergman R, Sprecher E, Schoenau E (2005) A novel homozygous missense mutation in FGF23 causes familial tumoral calcinosis associated with disseminated visceral calcification. Hum Genet 118:261266

Frishberg Y, Topaz O, Bergman R, Behar D, Fisher D, Gordon D, Richard G, Sprecher E (2005) Identification of a recurrent mutation in GALNT3 demonstrates that hyperostosis-hyperphosphatemia syndrome and familial tumoral calcinosis are allelic disorders. J Mol Med 83:33-38

Fukumoto S (2005) Post-translational modification of fibroblast growth factor 23. Ther Apher Dial 9:319-322

Gal G, Metzker A, Garlick J, Gold Y, Calderon S (1994) Head and neck manifestations of tumoral calcinosis. Oral Surg Oral Med Oral Pathol 77:158-166

Hawass N el-D, Kolawole T, Ismail AH, Patel PJ (1988) Tumoral calcinosis: case reports from Saudi Arabia with a review of the literature. Trop Geogr Med 40:58-63

Ichikawa S, Lyles KW, Econs MJ (2005) A novel GALNT3 mutation in a pseudo-autosomal dominant form of tumoral calcinosis: evidence that the disorder is autosomal recessive. J Clin Endocrinol Metab 90:2420-2423

Inoue Y, Segawa H, Kaneko I, Yamanaka S, Kusano K, Kawakami E, Furutani J, Ito M, Kuwahata M, Saito H, Fukushima N, Kato S, Kanayama HO, Miyamoto K (2005) Role of the vitamin D receptor in FGF23 action on phosphate metabolism. Biochem J 390:325-331

Jain SP (1989) Tumoral calcinosis in Somalia and Ethiopia: a report of twenty one cases and brief review of literature. East Afr Med J 66:476-480
Larsson T, Yu X, Davis SI, Draman MS, Mooney SD, Cullen MJ, White KE (2005) A novel recessive mutation in Fibroblast growth factor-23 (FGF23) causes familial tumoral calcinosis. J Clin Endocrinol Metab 90:2424-2427

McClatchie S, Bremner AD (1969) Tumoural calcinosis-an unrecognized disease. Br Med J 1:153-155

Metzker A, Eisenstein B, Oren J, Samuel R (1988) Tumoral calcinosis revisited - common and uncommon features. Report of ten cases and review. Eur J Pediatr 147:128-132

Miyamoto K, Ito M, Kuwahata M, Kato S, Segawa H (2005) Inhibition of intestinal sodium-dependent inorganic phosphate transport by fibroblast growth factor 23. Ther Apher Dial 9:331-335

Saito H, Kusano K, Kinosaki M, Ito H, Hirata M, Segawa H, Miyamoto K, Fukushima N (2003) Human fibroblast growth factor-23 mutants suppress $\mathrm{Na}+$-dependent phosphate cotransport activity and 1alpha,25-dihydroxyvitamin D3 production. J Biol Chem 278:2206-2211

Shimada T, Yamazaki Y, Takahashi M, Hasegawa H, Urakawa I, Oshima T, Ono K, Kakitani M, Tomizuka K, Fujita T, Fukumoto S, Yamashita T (2005) Vitamin D receptor-independent FGF23 actions in regulating phosphate and vitamin D metabolism. Am J Physiol Renal Physiol 289:F1088-F1095

Smack D, Norton SA, Fitzpatrick JE (1996) Proposal for a pathogenesis-based classification of tumoral calcinosis. Int J Dermatol 35:265-271

Ten Hagen KG, Fritz TA, Tabak LA (2003) All in the family: the UDP-GalNAc:polypeptide $N$-acetylgalactosaminyltransferases. Glycobiology 13:1R-16R

Topaz O, Shurman D, Bergman R, Indelman M, Ratajczak P, Mizrachi M, Khamaysi Z, Behar D, Petronius D, Friedman V, Zelikovic I, Raimer S, Metzker A, Richard G, Sprecher E (2004) Mutations in GALNT3, encoding a protein involved in O-linked glycosylation, cause familial tumoral calcinosis. Nat Genet 36:579-581

Vogt G, Chapgier A, Yang K, Chuzhanova N, Feinberg J, Fieschi C, Boisson-Dupuis S, Alcais A, Filipe-Santos O, Bustamante J, de Beaucoudrey L, Al-Mohsen I, Al-Hajjar S, Al-Ghonaium A, Adimi P, Mirsaeidi M, Khalilzadeh S, Rosenzweig S, de la Calle Martin O, Bauer TR, Puck JM, Ochs HD, Furthner D, Engelhorn C, Belohradsky B, Mansouri D, Holland SM, Schreiber RD, Abel L, Cooper DN, Soudais C, Casanova JL (2005) Gains of glycosylation comprise an unexpectedly large group of pathogenic mutations. Nat Genet 37:692-700

$\mathrm{Yu}$ X, Ibrahimi OA, Goetz R, Zhang F, Davis SI, Garringer HJ, Linhardt RJ, Ornitz DM, Mohammadi M, White KE (2005) Analysis of the biochemical mechanisms for the endocrine actions of fibroblast growth factor-23. Endocrinology $146: 4647-4656$ 